\title{
Isoperimetric Problems for Convex Bodies and a Localization Lemma*
}

\author{
R. Kannan, ${ }^{1}$ L. Lovász, ${ }^{2}$ and M. Simonovits ${ }^{3}$ \\ ${ }^{1}$ Department of Computer Science, Carnegie-Mellon University, \\ Pittsburgh, PA 15213, USA \\ 2 Department of Computer Science, Yale University, \\ New Haven, CT 06520, USA \\ ${ }^{3}$ Mathematical Institute, Hungarian Academy of Sciences, \\ Reáltanoda u. 13-15, H·1053 Budapest, Hungary
}

\begin{abstract}
We study the smallest number $\psi(K)$ such that a given convex body $K$ in $\mathbb{R}^{n}$ can be cut into two parts $K_{1}$ and $K_{2}$ by a surface with an $(n-1)$-dimensional measure $\psi(K) \operatorname{vol}\left(K_{1}\right) \cdot \operatorname{vol}\left(K_{2}\right) / \operatorname{vol}(K)$. Let $M_{1}(K)$ be the average distance of a point of $K$ from its center of gravity. We prove for the "isoperimetric coefficient" that

$$
\psi(K) \geq \frac{\ln 2}{M_{1}(K)},
$$

and give other upper and lower bounds. We conjecture that our upper bound is the exact value up to a constant.

Our main tool is a general "Localization Lemma" that reduces integral inequalities over the $n$-dimensional space to integral inequalities in a single variable. This lemma was first proved by two of the authors in an earlier paper, but here we give various extensions and variants that make its application smoother. We illustrate the usefulness of the lemma by showing how a number of well-known results can be proved using it.
\end{abstract}

* The work of R. Kannan was done while visiting Yale University and was supported by NSF Grant CCR-9208597 to Carnegie-Mellon. The work of László Lovász was supported by NSF Grant CCR-9402916. 


\section{Isoperimetry in a Convex Body}

The classical isoperimetric problem is to find a surface with minimal measure which encloses a set of (at least) a given volume. We consider a "relativized" version of this problem, where we are given a convex body $K$, and want to find a surface which divides $K$ into two parts, and whose measure is minimum relative to the volumes of the two parts.

To be more precise:

(a) The ( $n-1)$-dimensional Minkowski measure of a set $A \subseteq \mathbb{R}^{n}$ is defined as the limit (if it exists) of the volume of the $\varepsilon / 2$-neighborhood of $A$ divided by $\varepsilon$, when $\varepsilon \rightarrow 0$. (Volume means Lebesgue measure.)

Define the isoperimetric coefficient of a convex body $K \subseteq \mathbb{R}^{n}$ as the largest number $\psi=\psi(K)$ such that, for every measurable subset $S \subseteq K$ for which $\partial_{K} S$ (the boundary of $S$, relative to $K$ ) has an $(n-1)$-dimensional Minkowski measure,

$$
\operatorname{vol}_{n-1}\left(\partial_{K} S\right) \geq \psi \frac{\operatorname{vol}(S) \cdot \operatorname{vol}(K \backslash S)}{\operatorname{vol}(K)} .
$$

Inequality (1.1) is often replaced in the definition by

$$
\operatorname{vol}_{n-1}\left(\partial_{K} S\right) \geq \psi \min \{\operatorname{vol}(S), \operatorname{vol}(K \backslash S)\}
$$

Since the two values are always within a factor of 2 to each other, this does not influence our results in any essential way. Our formulation is more natural when applying the results to Markov chains. Lower bounds on $\psi$ were proved by Bokowski and Spencer (1979) and Bokowski (1980).

The problem of finding sharp lower bounds on the isoperimetric coefficient arose (among others) in connection with randomized volume algorithms. Dyer et al. (1989) formulated this connection and conjectured a lower bound on $\psi$. Karzanov and Khachiyan (1991) and Lovász and Simonovits (1990) proved (by different methods) a lower bound of $1 / d$ (where $d$ is the diameter of $K$ and the conductance was defined according to (1.2)). This result was generalized to the case when Lebesgue measure is replaced by any measure given by a log-concave function $F$ (i.e., when $\operatorname{vol}(T)$ is replaced by $\int_{T} F$ ) by Applegate and Kannan (1990). The bound (still using (1.2)) was improved to $\psi(K) \geq 2 / d$ by Dyer and Frieze (1992), which is the best possible bound (even for Lebesgue measure) in terms of the diameter, as shown by a very thin long cylinder.

However, the diameter may not be the best possible measure to use here. In its applications to volume algorithms and related questions, we need these bounds in the case when the body is rather "round" (e.g., in isotropic position, see below), and hence the bound $2 / d$ may not be sharp. In this paper we prove two improvements of this bound, in terms of somewhat more complicated measures of the "linear dimensions" of $K$. In particular, denote the average distance of a point in $K$ from its center of gravity by $M_{1}(K)$. Note that $M_{1}(K)$ may be much smaller than the diameter, e.g., in case of a regular simplex $S^{n}$ of diameter $d, M_{1}\left(S^{n}\right) \approx d / \sqrt{n}$. 
Main Theorem. For every convex body $K$,

$$
\psi(K) \geq \frac{\ln 2}{M_{1}(K)} .
$$

We also formulate a conjecture that would determine the value of the isoperimetric coefficient up to a constant factor.

We start by describing a general method for proving inequalities involving the volume and other measures (Localization Lemma). This method reduces highdimensional integral inequalities to one-dimensional ones. In Section 3 we illustrate the method by giving new proofs of some important inequalities in convex geometry, some of which are sharper than the earlier versions. Our results on the isoperimetric problem are contained in Section 5.

\section{Localization Lemma}

Below we use the notion of upper and lower semicontinuous functions.

Lower semicontinuous functions are limits of monotone increasing sequences of continuous functions. They can also be characterized by that

$$
\liminf _{x \rightarrow x_{0}} f(x)=f\left(x_{0}\right) \quad \text { for every } \quad x \in D_{f},
$$

where $D_{f}$ is the domain of $f$, in our case mostly an open or closed convex set $K$ or the whole space $\mathbb{R}^{n}$. The indicator function of an open set is always lower semicontinuous but not continuous. This is the reason why we cannot restrict our considerations to continuous functions. Upper semicontinuous functions are the limits of monotone decreasing functions, and the indicator functions of closed sets are upper semicontinuous.

Corrigendum to the Lovász-Simonovits Paper (1993). In our earlier paper we exchanged the word "upper" and "lower" in speaking of semicontinuous functions. Otherwise everything was correct. Here we return to the usual (correct) definitions.

The following general tool for proving geometric inequalities was proved by Lovász and Simonovits (1993).

Lemma 2.1. Let $g$ and $h$ be lower semicontinuous Lebesgue integrable functions on $\mathbb{R}^{n}$ such that

$$
\int_{\mathbb{R}^{n}} g(x) d x>0 \text { and } \int_{\mathbb{R}^{n}} h(x) d x>0
$$

Then two points $a, b \in \mathbb{R}^{n}$ and a linear function $l:[0,1] \rightarrow \mathbb{R}_{+}$exist such that

$$
\int_{0}^{1} l(t)^{n-1} g((1-t) a+t b) d t>0 \text { and } \int_{0}^{1} l(t)^{n-1} h((1-t) a+t b) d t>0
$$


To formulate the conclusion informally, we may consider $(l(t))^{n-1} d A$ to be the cross-sectional area $((n-1)$ dimensional volume) of an infinitesimal cone with base area $d A$; then the conclusion of the lemma just states that the integrals of both $g$ and $h$ over this cone truncated at $a, b$ are positive.

In fact, it is convenient to make this precise by introducing the following language. By a needle we mean a segment $I=[a, b]$ in $\mathbb{R}^{n}$, together with a nonnegative linear function $l: I \rightarrow \mathbb{R}_{+}$not identically 0 . If $N=(I, l)$ is a needle and $f$ is an integrable function defined on $I$, then we set

$$
\int_{N} f=\int_{0}^{|b-a|} f(a+t u) l(a+t u)^{n-1} d t
$$

where $u=(1 / \mid b-a)(b-a)$.

It is easy to show that we could not restrict the family of these "test bodies" further - in Lemma 2.1 - to (untruncated) infinitesimally narrow cones, or cylinders (i.e., choosing $l$ to be constant, or zero at one of the endpoints). Furthermore, this theorem cannot be directly generalized to three integrals. (If the sign of $k$ integrals is to be preserved, then $(k-1)$-dimensional test-bodies have to be used. We do not elaborate on this idea here.)

We state two corollaries of this lemma which are sometimes more convenient to apply.

Corollary 2.2. Let $f_{1}, f_{2}, f_{3}, f_{4}$ be four nonnegative continuous functions defined on $\mathbb{R}^{n}$, and $\alpha, \beta>0$. Then the following are equivalent:

(a) For every convex body $K$ in $\mathbb{R}^{n}$,

$$
\left(\int_{K} f_{1}\right)^{\alpha}\left(\int_{K} f_{2}\right)^{\beta} \leq\left(\int_{K} f_{3}\right)^{\alpha}\left(\int_{K} f_{4}\right)^{\beta} .
$$

(b) For every needle $N$ in $\mathbb{R}^{n}$,

$$
\left(\int_{N} f_{1}\right)^{\alpha}\left(\int_{N} f_{2}\right)^{\beta} \leq\left(\int_{N} f_{3}\right)^{\alpha}\left(\int_{N} f_{4}\right)^{\beta}
$$

Proof of Corollary 2.2. It is clear that (a) implies (b). To prove the converse, assume (b) for a convex body $K$ and assume indirectly that

$$
\left(\int_{K} f_{1}\right)^{\alpha}\left(\int_{K} f_{2}\right)^{\beta}>\left(\int_{K} f_{3}\right)^{\alpha}\left(\int_{K} f_{4}\right)^{\beta}
$$

By adding a small constant to $f_{3}$ and $f_{4}$, we may assume that they are positive and (2.1) still holds. We may also assume that $\int_{K} f_{i}>0$ for $i=1,2,3,4$. 
Fix an $A$ such that

$$
\frac{\left(\int_{K} f_{1}\right)^{\alpha}}{\left(\int_{K} f_{3}\right)^{\alpha}}>A>\frac{\left(\int_{K} f_{4}\right)^{\beta}}{\left(\int_{K} f_{2}\right)^{\beta}}>0 .
$$

Then

$$
\int_{K}\left(A^{1 / \beta} f_{2}-f_{4}\right)>0 \text { and } \int_{K}\left(f_{1}-A^{1 / \alpha} f_{3}\right)>0 .
$$

By the Localization Lemma, we have a needle $N$ contained in $K$ such that

$$
\int_{N}\left(A^{1 / \beta} f_{2}-f_{4}\right)>0 \text { and } \int_{N}\left(f_{1}-A^{1 / \alpha} f_{3}\right)>0
$$

These inequalities imply that $\int_{N} f_{1}>0$ and $\int_{N} f_{2}>0$. Clearly,

$$
\left(\int_{N} f_{1}\right)^{\alpha}\left(\int_{N} f_{2}\right)^{\beta}>A\left(\int_{N} f_{3}\right)^{\alpha}\left(\int_{N} f_{2}\right)^{\beta}>\left(\int_{N} f_{3}\right)^{\alpha}\left(\int_{N} f_{4}\right)^{\beta},
$$

contradicting assumption (b). ${ }^{1}$

Remark 2.3. It is easy to see that Corollary 2.2 automatically extends to the more general case when $f_{1}, f_{2}$ are upper semicontinuous and $f_{3}, f_{4}$ are lower semicontinuous functions. Indeed, then we may choose four sequences of continuous functions, $f_{i}^{(k)}$ so that $f_{1}^{(k)} \nearrow f_{1}, f_{2}^{(k)} \nearrow f_{2}, f_{3}^{(k)} \searrow f_{3}, f_{4}^{(k)} \searrow f_{4}$ : (b) will be trivially satisfied by the approximating functions. If (a) were violated by $f_{1}, f_{2}, f_{3}, f_{4}$, then for some sufficiently large $k$ the continuous $f_{1}^{(k)}, f_{2}^{(k)}, f_{3}^{(k)}, f_{4}^{(k)}$ would also violate it, contradicting the original corollary (continuous case). This observation is useful when we have functions defined and continuous on a subset $T$ of $\mathbb{R}^{n}$ and want to use the above arguments: we may extend these functions to the whole space by defining those outside to be zero. This continuous functions extend to upper semicontinuous functions if $T$ is closed, to lower semicontinuous ones if $T$ is open.

Corollary 2.4. Suppose $T$ is a bounded open convex set in $\mathbb{R}^{n}, g$ is a bounded lower semicontinuous function on $T$, and $h$ is a continuous function on $T$ such that

$$
\int_{T} g(x) d x>0 \text { and } \int_{T} h(x) d x=0 .
$$

Then a needle $N=(I, l)$ with $I \subseteq T$ exists such that

$$
\int_{N} g>0 \text { and } \int_{N} h=0
$$

\footnotetext{
${ }^{1}$ The fact that we possibly added a small positive constant to $f_{3}$ and $f_{4}$ works in the right direction: we get a contradiction with the original $f_{3}, f_{4}$, too.
} 
Remark 2.5. It is clear that we can replace strict inequality by $\geq$ in the assumption and in the conclusion.

Proof. This assertion could be proved along the lines of the original proof of Lemma 2.1 (Lemma 2.5 in Lovász and Simonovits (1993)) but - using a simple trick -it can also be reduced to Lemma 2.1 as follows.

Choose a $\delta>0$ such that $\int_{T}(g-\delta)>0$. Let $\varepsilon$ be any positive real (which will later tend to zero). We have

$$
\int_{T}\left(g-\delta+\frac{1}{\varepsilon} h\right)>0 \text { and } \int_{T}\left(\varepsilon^{2}-h\right)>0
$$

We convert the above integrals over $T$ into integrals over $\mathbb{R}^{n}$ by multiplying the integrand by the (lower semicontinuous) indicator function of $T$ and then we apply Lemma 2.1. Thus we get a needle $N_{\varepsilon}$ for which

$$
\int_{N_{\varepsilon}}\left(g-\delta+\frac{1}{\varepsilon} h\right)>0 \text { and } \int_{N_{\varepsilon}}\left(\varepsilon^{2}-h\right)>0 \text {. }
$$

Hence

$$
\int_{N_{\varepsilon}}(g-\delta+\varepsilon)>0
$$

and if $M$ is an upper bound on $g$ on all of $T$, then

$$
\int_{N_{\varepsilon}} h>\int_{N_{\varepsilon}}(-\varepsilon g) \geq-M \varepsilon \int_{N_{\varepsilon}} 1 \text { and } \int_{N_{\varepsilon}} h<\varepsilon^{2} \int_{N_{\varepsilon}} 1
$$

We construct these needles $N_{\varepsilon}$ for $\varepsilon=1 / k$ for $k=1,2,3, \ldots$ We may assume after scaling by a suitable positive real that the maximum of each linear function corresponding to the needles is 1 . There is then a subsequence of these needles that converges (in the sense that the intervals converge to an interval and the linear functions to some linear function with maximum $=1$ ). It is easy to see (using the continuity of $h$ ) that, for the "limit" needle intersected by $T$, the conclusions are valid.

Next we prove a version of Corollary 2.2 involving log-concave weight functions. Here exponential needles, defined below, replace the needles. However, even if we are not concerned with log-concave functions, exponential needles are easier to use. The Localization Lemma (or its corollary stated above) reduces the problem of proving inequalities between integrals in $\mathbb{R}^{n}$ to inequalities between onedimensional integrals. However, then $n$th powers of linear functions in the definition of a "needle" are often difficult to handle. In the case when we want to prove inequalities that are independent of $n$ (in some sense), the following theorem may 
be easier to apply. To state it, we define an exponential needle $E$ as a segment $[a, b] \subseteq \mathbb{R}^{n}$, together with a real constant $\gamma$; and we define the integral over such a needle by

$$
\int_{E} f=\int_{0}^{|b-a|} f(a+t u) e^{\gamma t} d t
$$

where $u=(1 /|b-a|)(b-a)$.

Recall that a function $f: \mathbb{R}^{n} \rightarrow \mathbb{R}_{+}$is log-concave if it satisfies, for all $x, y \in \mathbb{R}^{n}$ and $0<\lambda<1$,

$$
f(\lambda x+(1-\lambda) y) \geq f(x)^{\lambda} f(y)^{(1-\lambda)} .
$$

Equivalently, a (nonnegative) function is log-concave if its support

$$
K=\left\{x \in \mathbb{R}^{n}: f(x)>0\right\}
$$

is convex, and $\log f$ is a concave function on $K$. Every nonnegative function that is concave over a convex domain is log-concave (we define its value to be zero outside the original domain). In particular, the indicator function of a convex body is log-concave. Log-concave functions include many density functions important in statistics, e.g., $e^{-x^{2}}$ and $e^{-|x|}$. If $l$ is a linear function, then $\max \left\{0, l^{k}\right\}$ is log-concave.

We need a lemma about the one-dimensional case.

Lemma 2.6. Let $f_{1}, f_{2}, f_{3}, f_{4}$ be four nonnegative continuous functions defined on an interval $[a, b]$, and $\alpha, \beta>0$. Then the following are equivalent:

(a) For every log-concave function $F$ defined on $\mathbb{R}$,

$$
\left(\int_{a}^{b} F(t) f_{1}(t) d t\right)^{\alpha}\left(\int_{a}^{b} F(t) f_{2}(t) d t\right)^{\beta} \leq\left(\int_{a}^{b} F(t) f_{3}(t) d t\right)^{\alpha}\left(\int_{a}^{b} F(t) f_{4}(t) d t\right)^{\beta}
$$

(b) For every subintenval $\left[a^{\prime}, b^{\prime}\right] \subseteq[a, b]$, and every real $\gamma$,

$$
\left(\int_{a^{\prime}}^{b^{\prime}} e^{\gamma t} f_{1}(t) d t\right)^{\alpha}\left(\int_{a^{\prime}}^{b^{\prime}} e^{\gamma t} f_{2}(t) d t\right)^{\beta} \leq\left(\int_{a^{\prime}}^{b^{\prime}} e^{\gamma t} f_{3}(t) d t\right)^{\alpha}\left(\int_{a^{\prime}}^{b^{\prime}} e^{\gamma t} f_{4}(t) d t\right)^{\beta}
$$

Proof. First we note that if a point $t_{0} \in[a, b]$ exists such that $\left(f_{1}\left(t_{0}\right)\right)^{\alpha}\left(f_{2}\left(t_{0}\right)\right)^{\beta}>$ $\left(f_{3}\left(t_{0}\right)\right)^{\alpha}\left(f_{4}\left(t_{0}\right)\right)^{\beta}$, then both assertions fail (by considering a very small interval containing $t_{0}$, or the log-concave function $e^{-C\left(t-t_{0}\right)^{2}}$ with a large constant $C$ ). So we may assume that, for all $a \leq t \leq b$,

$$
f_{1}(t)^{\alpha} f_{2}(t)^{\beta} \leq f_{3}(t)^{\alpha} f_{4}(t)^{\beta} \text {. }
$$

It is obvious that (a) implies (b).

(b) $\Rightarrow$ (a). Assume that (a) fails for some log-concave function $F$. We may assume that $F(t)>0$ for all $a \leq t \leq b$, since replacing $F$ by, say, its convolution with $e^{-C t^{2}}$ 
with a very large $C$ would still yield a counterexample: indeed, the convolution of log-concave functions is again log-concave (see Dinghas, 1957; Prékopa, 1971). By scaling, we may also assume that $F(t) \geq 1$ on $[a, b]$. So we can write $F=e^{G}$, where $G$ is a nonnegative concave function on $[a, b]$.

Also, we may choose an $\varepsilon>0$ sufficiently small such that

$$
\begin{aligned}
& \left(\int_{a}^{b} F(t) f_{1}(t) d t\right)^{\alpha}\left(\int_{a}^{b} F(t) f_{2}(t) d t\right)^{\beta} \\
& \quad>\left(\int_{a}^{b} F(t)\left(f_{3}(t)+\varepsilon\right) d t\right)^{\alpha}\left(\int_{a}^{b} F(t)\left(f_{4}(t)+\varepsilon\right) d t\right)^{\beta} .
\end{aligned}
$$

For each natural number $n$, consider the convex body $K_{n}$ below in $\mathbb{R}^{n+1}$ :

$$
K_{n}=\left\{(t, x): t \in[a, b], x \in \mathbb{R}^{n},\|x\| \leq 1+\frac{G(t)}{n}\right\}
$$

We also extend each $f_{i}$ to a function $\hat{f}_{i}: \mathbb{R}^{n+1} \rightarrow \mathbb{R}$ by $\hat{f}_{i}(t, x)=f_{i}(t)$.

Then, for sufficiently large $n$, we have $(1+G(t) / n)^{n} \approx e^{G(t)}$ and (2.4) implies

$$
\left(\int_{K_{n}} \hat{f}_{1}\right)^{\alpha}\left(\int_{K_{n}} \hat{f}_{2}\right)^{\beta}>\left(\int_{K_{n}}\left(\hat{f_{3}}+\varepsilon\right)\right)^{\alpha}\left(\int_{K_{n}}\left(\hat{f_{4}}+\varepsilon\right)\right)^{\beta} \text {. }
$$

By Corollary 2.2, a needle $N_{n}=\left(I_{n}, l_{n}\right)$ in $\mathbb{R}^{n+1}$ exists such that

$$
\left(\int_{N_{n}} \hat{f}_{1}\right)^{\alpha}\left(\int_{N_{n}} \hat{f}_{2}\right)^{\beta}>\left(\int_{N_{n}}\left(\hat{f_{3}}+\varepsilon\right)\right)^{\alpha}\left(\int_{N_{n}}\left(\hat{f_{4}}+\varepsilon\right)\right)^{\beta}
$$

If the needle is orthogonal to the $t$-axis, then this inequality violates (2.3), so we assume this is not the case. Thus, we may project to $[a, b]$ and assume that $I_{n}=\left[a_{n}, b_{n}\right] \subseteq[a, b]$ and that $l_{n}$ is a linear function of $t \in\left[a_{n}, b_{n}\right]$, so we have

$$
\begin{aligned}
& \left(\int_{a_{n}}^{b_{n}} l_{n}(t)^{n} f_{1}(t) d t\right)^{\alpha}\left(\int_{a_{n}}^{b_{n}} l_{n}(t)^{n} f_{2}(t) d t\right)^{\beta} \\
& \quad>\left(\int_{a_{n}}^{b_{n}} l_{n}(t)^{n}\left(f_{3}(t)+\varepsilon\right) d t\right)^{\alpha}\left(\int_{a_{n}}^{b_{n}} l_{n}(t)^{n}\left(f_{4}(t)+\varepsilon\right) d t\right)^{\beta} .
\end{aligned}
$$

There is a subsequence $(n)$ such that in the subsequence, $a_{n} \rightarrow a_{0}$ and $b_{n} \rightarrow b_{0}$. Inequality (2.3) implies that $a_{0}<b_{0}$. We may assume that $l_{n}\left(a_{0}\right) \leq l_{n}\left(b_{0}\right)$ for infinitely many indices (otherwise, we may exchange $a_{0}$ and $b_{0}$ for the argument below). We may also assume that each $l_{n}$ is normalized so that $l_{n}\left(b_{0}\right)=1$. Let $\gamma_{n}=l_{n}\left(a_{0}\right)$. We may also assume that $\gamma_{n} \rightarrow \gamma$ and $\left(1-\gamma_{n}\right) n \rightarrow \gamma^{\prime}$ for some $0 \leq \gamma$ $\leq 1$ and $0 \leq \gamma^{\prime} \leq \infty$, for an infinite sequence of values of $n$. (Note: $\gamma^{\prime}$ may be infinity.) 
If $\gamma \neq 1$, then $l_{n}^{n} \rightarrow 0$ for every $a_{0} \leq t<b_{0}$. However, then, dividing both sides of (2.5) by $\left(\int_{a_{n}}^{b_{n}} l_{n}(t)^{n} d t\right) \alpha+\beta$ and letting $n \rightarrow \infty$, we get that

$$
f_{1}\left(b_{0}\right)^{\alpha} f_{2}\left(b_{0}\right)^{\beta} \geq\left(f_{3}\left(b_{0}\right)+\varepsilon\right)^{\alpha}\left(f_{4}\left(b_{0}\right)+\varepsilon\right)^{\beta},
$$

which contradicts (2.3).

So we have $\gamma=1$. Then $l_{n}(t) \rightarrow 1$ and so we have, for each $a_{0} \leq t \leq b_{0}$,

$$
l_{n}(t)^{n}=\left(1-\left(1-l_{n}(t)\right)\right)^{n}=\left[\left(1-\left(1-l_{n}(t)\right)\right)^{1 /\left(1-l_{n}(t)\right)}\right]^{n\left(1-l_{n}(t)\right)} .
$$

Here the base (in the square brackets) tends to $1 / e$. If $\gamma^{\prime}=\infty$, then $l_{n}(t)^{n} \rightarrow 0$ for $t<b_{0}$, which leads to a contradiction as before. If $\gamma^{\prime}$ is finite, then (using that $l_{n}$ is linear) we have $n\left(1-l_{n}(t)\right) \rightarrow \gamma^{\prime}\left(b_{0}-t\right) /\left(b_{0}-a_{0}\right)$, and so

$$
l_{n}(t)^{n} \rightarrow e^{\gamma^{\prime}\left(t-b_{0}\right) /\left(b_{0}-a_{0}\right)}
$$

Thus it follows that (with $\gamma^{\prime \prime}=\gamma^{\prime} /\left(b_{0}-a_{0}\right)$ )

$$
\begin{aligned}
& \left(\int_{a_{0}}^{b_{0}} e^{\gamma^{\prime \prime}\left(t-b_{0}\right)} f_{1}(t) d t\right)^{\alpha}\left(\int_{a_{0}}^{b_{0}} e^{\gamma^{\prime \prime}\left(t-b_{0}\right)} f_{2}(t) d t\right)^{\beta} \\
& \quad \geq\left(\int_{a_{0}}^{b_{0}} e^{\gamma^{\prime \prime}\left(t-b_{0}\right)}\left(f_{3}(t)+\varepsilon\right) d t\right)^{\alpha}\left(\int_{a_{0}}^{b_{0}} e^{\gamma^{\prime \prime}\left(t-b_{0}\right)}\left(f_{4}(t)+\varepsilon\right) d t\right)^{\beta}
\end{aligned}
$$

and hence

$$
\left(\int_{a_{0}}^{b_{0}} e^{\gamma^{\prime \prime} t} f_{1}(t) d t\right)^{\alpha}\left(\int_{a_{0}}^{b_{0}} e^{\gamma^{\prime \prime} t} f_{2}(t) d t\right)^{\beta}>\left(\int_{a_{0}}^{b_{0}} e^{\gamma^{\prime \prime} t} f_{3}(t) d t\right)^{\alpha}\left(\int_{a_{0}}^{b_{0}} e^{\gamma^{\prime \prime} t} f_{4}(t) d t\right)^{\beta},
$$

showing that (b) is violated.

Theorem 2.7. Let $f_{1}, f_{2}, f_{3}, f_{4}$ be four nonnegative continuous functions defined on $\mathbb{R}^{n}$, and $\alpha, \beta>0$. Then the following are equivalent:

(a) For every log-concave function $F$ defined on $\mathbb{R}^{n}$ with compact support,

$$
\begin{aligned}
& \left(\int_{\mathbb{R}^{n}} F(t) f_{1}(t) d t\right)^{\alpha}\left(\int_{\mathbb{R}^{n}} F(t) f_{2}(t) d t\right)^{\beta} \\
& \quad \leq\left(\int_{\mathbb{R}^{n}} F(t) f_{3}(t) d t\right)^{\alpha}\left(\int_{\mathbb{R}^{n}} F(t) f_{4}(t) d t\right)^{\beta} .
\end{aligned}
$$

(b) For every exponential needle $E$,

$$
\left(\int_{E} f_{1}\right)^{\alpha}\left(\int_{E} f_{2}\right)^{\beta} \leq\left(\int_{E} f_{3}\right)^{\alpha}\left(\int_{E} f_{4}\right)^{\beta}
$$


Proof. The direction (a) $\Rightarrow$ (b) is easy: Let the exponential needle $E$ be defined by the segment $[a, b]\left(a, b \in \mathbb{R}^{n}\right)$ and constant $\gamma$, and let $u=(b-a) /|a-b|$ as before. Apply (a) to the $\log$-concave function $e^{\gamma u x}$, restricted to an $\varepsilon$-neighborhood of $[a, b]$, and let $\varepsilon \rightarrow 0$; we then get (b).

Conversely, assume that (b) holds and consider any log-concave function $F$. Assume that (a) fails to hold. Apply Corollary 2.2 to the functions $F f_{i}$; it follows that there is a segment $[a, b]$ and a linear function $l:[a, b] \rightarrow \mathbb{R}_{+}$such that

$$
\begin{aligned}
& \left(\int_{0}^{|b-a|} f_{1}(a+t u) F(a+t u) l(a+t u)^{n-1} d t\right)^{\alpha} \\
& \times\left(\int_{0}^{|b-a|} f_{2}(a+t u) F(a+t u) l(a+t u)^{n-1} d t\right)^{\beta} \\
& >\left(\int_{0}^{|b-a|} f_{3}(a+t u) F(a+t u) l(a+t u)^{n-1} d t\right)^{\alpha} \\
& \quad \times\left(\int_{0}^{|b-a|} f_{4}(a+t u) F(a+t u) l(a+t u)^{n-1} d t\right)^{\beta} .
\end{aligned}
$$

Now here $F l^{n-1}$ is also log-concave, and hence Lemma 2.4 implies that there is an exponential needle $E$ violating (b).

Remark 2.8. An exponential needle consists of an interval and an exponent $\gamma$. We may often rescale, reducing the general case to the case $\gamma=1$. The case $\gamma=0$ can be neglected since in our cases it always follows from the case $\gamma \neq 0$ by going to limits.

\section{Applications}

As an illustration of the use of the Localization Lemma, we derive a generalization of Khintchine's inequality, due to Gromov and Milman (1984); see also Milman and Pajor (1989). Other geometric inequalities, like the extension of Theorem 3.1 below to norms of polynomials of bounded degree (Bourgain, 1991), a spherical Brunn-Minkowski type theorem of Borell (1975), a theorem of Hensley (1980) on sections of isotropic bodies, or an inequality of Dinghas (1957) on log-concave functions can also be deduced using the Localization Lemma (see also Lovász and Simonovits, 1993). Typically, this method also enables the best constants in these results to be found, although (as is also illustrated below) at the cost of a tedious computation.

Let $K$ be a convex body and let $f: K \rightarrow \mathbb{R}$ be an integrable function. We define its $L_{p}$-norm by

$$
\|f\|_{p}=\left(\frac{1}{\operatorname{vol}(K)} \int_{K}|f(x)|^{p} d x\right)^{1 / p}
$$


If $0<p<q$, then clearly $\|f\|_{p} \leq\|f\|_{q}$; the next theorem asserts that if $f$ is linear, then the following converse also holds:

Theorem 3.1. A constant $c_{p, q}$ depending only on $p$ and $q$ (so independent of the dimension and of $K$ ) exists such that, for every convex body $K$ in $\mathbb{R}^{n}$ and every linear function $f: K \rightarrow \mathbb{R}$,

$$
\|f\|_{q} \leq c_{p, q}\|f\|_{p} .
$$

Proof. We write inequality (3.1) in the following form:

$$
\left(\int_{K}|f|^{q}\right)^{1 / q}\left(\int_{K} 1\right)^{1 / p} \leq c_{p, q}\left(\int_{K}|f|^{p}\right)^{1 / p}\left(\int_{K} 1\right)^{1 / q} .
$$

By Theorem 2.7, it suffices to prove that, for every exponential needle $E$,

$$
\left(\int_{E}|f|^{q}\right)^{1 / q}\left(\int_{E} 1\right)^{1 / p} \leq c_{p, q}\left(\int_{E}|f|^{p}\right)^{1 / p}\left(\int_{E} 1\right)^{1 / q} .
$$

Translating this into one-dimensional integrals, we must prove (for every fixed $0<p<q$ ) that a constant $c_{p, q}$ exists such that, for every linear function $f$, every $a<b$, and every real $\gamma$,

$$
\left(\frac{\int_{a}^{b} e^{\gamma t}|f(t)|^{q} d t}{\int_{a}^{b} e^{\gamma t} d t}\right)^{1 / q} \leq c_{p, q}\left(\frac{\int_{a}^{b} e^{\gamma t}|f(t)|^{p} d t}{\int_{a}^{b} e^{\gamma t} d t}\right)^{1 / p} .
$$

Without loss of generality, we may assume that $f(t)=t$ and $\gamma=1$ (this implies the case $\gamma \neq 0$ by substitution, and then the cases $\gamma=0$ and $f(t)=c$ also follow by going to the limits). Let

$$
\varphi(a, b):=\frac{\left(\int_{a}^{b} e^{t}|f(t)|^{q} d t / \int_{a}^{b} e^{t} d t\right)^{1 / q}}{\left(\int_{a}^{b} e^{t}|f(t)|^{p} d t / \int_{a}^{b} e^{t} d t\right)^{1 / p}}
$$

So we have $c_{p, q}=\sup _{a<b} \varphi(a, b)$, provided this is finite. However $\varphi(a, b)$ is continuous for $a<b$, and we can extend it to $a \leq b$ continuously, putting $\varphi(a, a)=1$ (since, for every $\alpha, \varphi(a, b)$ tends to 1 if $a<b$ and $a, b \rightarrow \alpha)^{2}$ and $\varphi(a, b) \rightarrow 1$ uniformly in $a$ if $b \rightarrow \infty$, and $a<b$ is arbitrary. Moreover, $\varphi(a, b)$ remains bounded if $b$ is bounded and $a \rightarrow-\infty$. This implies that $\varphi(a, b)$ remains bounded. To determine the best value of the constant, the supremum has to be found numerically, which is tedious but rather straightforward. For example, $c_{1,2}<2.5$ is obtained.

\footnotetext{
${ }^{2}$ The case $\alpha=0$ should be handled with some extra care.
} 


\section{Isotropy}

Given a convex body $K \subseteq \mathbb{R}^{n}$ and a function $f: K \rightarrow \mathbb{R}^{m}$, we denote by $E_{K}(f)$ the "average of $f$ over $K$," i.e.,

$$
E_{K}(f)=\frac{1}{\operatorname{vol}(K)} \int_{K} f(x) d x
$$

We denote by $b=b(K)=E_{K}(x)$ the center of gravity (baricenter) of $K$. We also let $A(K)$ denote the $n \times n$ matrix of "inertia" about $b(K)$ :

$$
E_{K}\left((x-b)(x-b)^{\mathrm{T}}\right),
$$

where superscript $\mathrm{T}$ denotes the transpose. The $p$ th moment $(p \geq 0)$ of a convex body $K$ is defined by

$$
M_{p}(K)=E_{K}\left(|x-b|^{p}\right)
$$

The second moment is just the trace of $A(K)$. It is easy to see that the average square distance between points in $K$ is $2 M_{2}(K)$ :

$$
\frac{1}{\operatorname{vol}(K)^{2}} \int_{K} \int_{K}|x-y|^{2} d x d y=2 M_{2}(K)
$$

It is clear that $M_{p}(K)^{(1 / p)}$ tends to $\max \{|x-b|: x \in K\}$ monotone increasing, as $p \rightarrow \infty$.

A body $K \subseteq \mathbb{R}^{n}$ is in isotropic position if $b(K)=0$ and $A(K)=I$, the identity matrix. Clearly, if $K$ is in isotropic position, then its second moment is $n$. (This definition is somewhat different from that in Milman and Pajor (1989), where it is assumed that $\operatorname{vol}(K)=1$ and $A(K)=\lambda_{K} I$. We have found our definition to be more convenient to use, in particular in probabilistic applications. It is known that $\lambda_{K}$ is bounded from below by an absolute constant, and it is a major conjecture that $\lambda_{K}$ is bounded from above by an absolute constant.)

We start with a lemma which is, in a sense, folklore. For centrally symmetric bodies, the corresponding result (in which case the bounds are somewhat sharper, but only by absolute constants) was proved by Milman and Pajor (1989). For the nonsymmetric case, the inequality, up to absolute constants, was proved by Sonnevend (1989).

Theorem 4.1. If $K$ is in isotropic position and $B$ is the unit ball about zero, then

$$
\sqrt{\frac{n+2}{n}} B \subseteq K \subseteq \sqrt{n(n+2)} B .
$$

(Note the slightly weaker but simpler inequalities $B \subseteq K \subseteq(n+1) B$. The inequalities as stated are tight for the regular simplex. Also, they imply the theorem of John (1948) on the inscribed and circumscribed ellipsoid of a convex body.) 
Proof. To prove the first containment, we use Corollary 2.4. Assume that $\sqrt{(n+2) / n} B \nsubseteq K$. Now, choosing the coordinate system appropriately, $K$ is contained in the half-space $x_{1}>-\sqrt{(n+2) / n}$. By hypothesis,

$$
\int_{K} x_{1}=0 \text { and } \int_{K}\left(x_{1}^{2}-1\right)=0
$$

Applying Remark 2.5 (extending Corollary 2.4) we get a needle $N=([a, b], l)$ contained in $K$, which we may assume is not orthogonal to the $x_{1}$ axis. Hence we may assume it is contained in the $x_{1}$ axis, such that

$$
\int_{a}^{b} x_{1}\left(l\left(x_{1}\right)\right)^{n-1}=0 \text { and } \int_{a}^{b} x_{1}^{2}\left(l\left(x_{1}\right)\right)^{n-1} \geq \int_{a}^{b}\left(l\left(x_{1}\right)\right)^{n-1}
$$

Our indirect hypothesis implies that $a>-\sqrt{(n+2) / n}$. It is easy to see that $l$ must be decreasing and then we may assume that $l(x)=t-x$ for some $t \geq b$. Then the integrals in both conditions can be evaluated explicitly, and a contradiction can be obtained by a tedious but rather straightforward computation.

The proof of the second inequality does not need the Localization Lemma. Let $v$ be the point of $K$ farthest from 0 . We have to show that $|v| \leq \sqrt{n(n+2)}$. Let $e=(1 /|v|) v$. For each $u \in \mathbb{R}^{n}$ with $|u|=1$, let $\phi(u)$ denote the largest real number $t$ with $v+t u \in K$. Then we have

$$
\operatorname{vol}(K)=\int_{\partial B} \int_{0}^{\phi(u)} t^{n-1} d t d u=\frac{1}{n} \int_{\partial B} \phi(u)^{n} d u
$$

Moreover,

$$
\begin{aligned}
1 & =\frac{1}{\operatorname{vol}(K)} \int_{K}\left(e^{T} x\right)^{2} d x=\frac{1}{\operatorname{vol}(K)} \int_{\partial B} \int_{0}^{\phi(u)} t^{n-1}\left(e^{T}(v+t u)\right)^{2} d t d u \\
& =\frac{1}{\operatorname{vol}(K)} \int_{\partial B}\left(\frac{\phi(u)^{n}}{n}|v|^{2}+2 \frac{\phi(u)^{n+1}}{n+1}|v| e^{T} u+\frac{\phi(u)^{n+2}}{n+2}\left(e^{T} u\right)^{2}\right) d u
\end{aligned}
$$

The integrand can be written as

$$
\frac{\phi(u)^{n}}{n}\left(\sqrt{\frac{n}{n+2}} \phi(u) e^{T} u+\frac{\sqrt{n(n+2)}}{n+1}|v|\right)^{2}+\frac{1}{n(n+1)^{2}} \phi(u)^{n}|v|^{2} .
$$

Here the first term is nonnegative, which gives the inequality

$$
1 \geq \frac{1}{\operatorname{vol}(K)} \int_{\partial B} \frac{1}{n(n+1)^{2}} \phi(u)^{n}|v|^{2}=\frac{|v|^{2}}{(n+1)^{2}},
$$


and hence $|v| \leq n+1$. To get the slightly stronger right-hand side inequality of (4.1), we give a positive lower bound on the first term in (4.2). Similarly as above, we have

$$
0=b(K)=\frac{1}{\operatorname{vol}(K)} \int_{\partial B} \int_{0}^{\phi(u)} t^{n-1}(v+t u) d t d u=v+\frac{1}{\operatorname{vol}(K)} \int_{\partial B} \frac{\phi(u)^{n+1}}{n+1} u d u
$$

and hence

$$
\begin{aligned}
& \frac{1}{\operatorname{vol}(K)} \int_{\partial B} \frac{\phi(u)^{n}}{n}\left(\sqrt{\frac{n}{n+2}} \phi(u) e^{T} u+\frac{\sqrt{n(n+2)}}{n+1}|v|\right) d u \\
& \quad=-\frac{1}{(n+1) \sqrt{n(n+2)}}|v| .
\end{aligned}
$$

Thus, by Cauchy-Schwarz,

$$
\begin{aligned}
& \frac{1}{\operatorname{vol}(K)} \int_{\partial B} \frac{\phi(u)^{n}}{n}\left(\sqrt{\frac{n}{n+2}} \phi(u) e^{T} u+\frac{\sqrt{n(n+2)}}{n+1}|v|\right)^{2} d u \\
& \geq \frac{1}{n(n+2)(n+1)^{2}}|v|^{2} .
\end{aligned}
$$

Thus

$$
1 \geq\left(\frac{1}{n(n+2)(n+1)^{2}}+\frac{1}{(n+1)^{2}}\right)|v|^{2}=\frac{1}{n(n+2)}|v|^{2},
$$

which proves the assertion.

\section{Isoperimetric Inequalities}

Now we state the main theorem of this paper.

Theorem 5.1. For every convex body $K$,

$$
\psi(K) \geq \frac{\ln 2}{M_{1}(K)} .
$$

Generally surfaces are difficult to handle. Therefore, in the definition of the isoperimetric coefficient, in (1.1), we replace the original surface $\partial S$ by its open $\varepsilon / 2$-neighborhood intersected by $K$, whose closure we denote by $K_{3}$. Further, we replace $S$ by $K_{1}:=S \backslash K_{3}$ and $K \backslash S$ by $K_{2}:=(K \backslash S) \backslash K_{3}$. So we prove the following theorem, which implies Theorem 5.1 by letting $\varepsilon \rightarrow 0$. 
Theorem 5.2. Let $K$ be a convex body and $K=K_{1} \cup K_{2} \cup K_{3}$, a decomposition of $K$ into three measurable sets such that the distance of $K_{1}$ and $K_{2}$ is $\varepsilon>0$. Then

$$
\operatorname{vol}\left(K_{1}\right) \operatorname{vol}\left(K_{2}\right) \leq \frac{M_{1}(K)}{\varepsilon \ln 2} \operatorname{vol}(K) \operatorname{vol}\left(K_{3}\right)
$$

Proof. The general case of measurable partition can be reduced to the case when $K_{1}$ and $K_{2}$ are closed: replacing $K$ by its closure we do not change vol $(K)$; replacing the original $K_{1}$ and $K_{2}$ by their closure we decrease neither vol $\left(K_{1}\right)$ nor $\operatorname{vol}\left(K_{2}\right)$; putting $K_{3}:=\bar{K} \backslash\left(\bar{K}_{1} \cup \bar{K}_{2}\right)$ we do not increase vol $\left(K_{3}\right)$. Hence Theorem 5.2 for this new partition will imply Theorem 5.2 for the old one.

We assume that $b(K)=0$. Let $f_{i}$ be the indicator function of $K_{i}(i=1,2,3)$ and $f_{4}(x)=|x| /(\varepsilon \ln 2)$. Then the assertion of the theorem is equivalent to

$$
\int_{K} f_{1} \int_{K} f_{2} \leq \int_{K} f_{3} \int_{K} f_{4}
$$

Theorem 2.7 can be applied and says that we only have to prove that

$$
\int_{E} f_{1} \int_{E} f_{2} \leq \int_{E} f_{3} \int_{E} f_{4}
$$

for every exponential needle $E$ contained in $K$.

Now let $E$ be defined by the segment $[a, b]$ and $\gamma$. We may assume that the segment intersects both $K_{1}$ and $K_{2}$. We may also assume that the origin corresponds to a point $u$ on the segment $[a, b]$, since otherwise we can move it to the nearest point on the segment and the function $f_{4}$ would only decrease while the other three would remain the same. We can rescale so that $\gamma=1$.

This reduces the problem to the following inequality concerning one-dimensional integrals. Let $a \leq 0 \leq b$ and $\gamma=1$. Let $[a, b]=J_{1} \cup J_{2} \cup J_{3}$ be a partition of $[a, b]$ into three measurable sets, so that the distance of $J_{1}$ and $J_{2}$ is at least $\varepsilon$. Then we want

$$
\int_{J_{1}} e^{t} d t \int_{J_{2}} e^{t} d t \leq \frac{1}{\varepsilon \ln 2} \int_{J_{3}} e^{t} d t \int_{a}^{b}|t-u| e^{t} d t
$$

We first prove the assertion in the (intuitively) most difficult case when $J_{1}, J_{2}$, and $J_{3}$ are intervals (naturally, $[c, d]=J_{3}$ is in the middle and has length at least $\varepsilon$ ). Dividing by $e^{c}$, we have to prove the inequality

$$
\int_{a}^{c} e^{t} d t \int_{e}^{b-c} e^{t} d t \leq \frac{1}{\varepsilon \ln 2} \int_{0}^{\varepsilon} e^{t} d t \int_{a}^{b}|t-u| e^{t} d t
$$

One way to prove this elementary inequality is to notice that the left-hand side is maximized when $c=(a+b-\varepsilon) / 2$, while the right-hand side is minimized when 
$u=\ln \left(\left(e^{a}+e^{b}\right) / 2\right)$. Substituting these values, and simplifying, we get that we have to prove

$$
\left(e^{(b-a) / 2}-e^{\varepsilon / 2}\right)^{2} \leq \frac{1}{\ln 2} \frac{e^{\varepsilon}-1}{\varepsilon}\left(-\ln \left(\frac{e^{a-b}+1}{2}\right) e^{b-a}+\ln \left(\frac{e^{b-a}+1}{2}\right)\right) .
$$

If we decrease $\varepsilon$, then the left-hand side increases while $\left(e^{\varepsilon}-1\right) / \varepsilon$ decreases, so it suffices to prove the inequality in the limit case $\varepsilon=0$. Substituting $z=e^{(b-a) / 2}$, we get that we have to prove

$$
(\ln 2)(z-1)^{2}+z^{2} \ln \left(\frac{z^{-2}+1}{2}\right)-\ln \left(\frac{z^{2}+1}{2}\right) \leq 0
$$

for $z \geq 1$. The function $f$ on the left-hand side satisfies

$$
\frac{d}{d z} \frac{f^{\prime}(z)}{z}=\frac{4\left(z^{3}-z\right)+2(\ln 2)\left(z^{2}+1\right)}{z^{2}\left(1+z^{2}\right)^{2}} \geq 0
$$

for $z \geq 1$, which implies that $f^{\prime}(z) / z$ is monotone increasing. Since $f^{\prime}(z) / z \rightarrow 0$ as $z \rightarrow \infty$, this implies that $f^{\prime}(z) \leq 0$, i.e., $f$ is monotone decreasing. Now since $f(1)=0$, this implies that $f(z) \leq 0$ for $z \geq 1$.

Thus we know that (5.1) holds when $J_{1}, J_{2}$, and $J_{3}$ are intervals. From this the assertion follows by a general trick from Lovász and Simonovits (1993). We may assume that $J_{3}$ is open, since replacing $J_{1}$ and $J_{2}$ by their closures would only make the inequality tighter. So $J_{3}$ is the union of disjoint open intervals; we may assume that these are of length at least $\varepsilon$, since the shorter intervals must have both endpoints in $J_{1}$ or both endpoints in $J_{2}$, and then accordingly we can add them to $J_{1}$ or $J_{2}$ and make the inequality tighter.

Now let $\left(c_{i}, d_{i}\right)(1 \leq i \leq k)$ be all maximal intervals contained in $J_{3}$. Then, by the argument above,

$$
\int_{a}^{c_{i}} e^{t} d t \int_{d_{i}}^{b} e^{t} d t \leq \frac{1}{\varepsilon \ln 2} \int_{c_{i}}^{d_{i}} e^{t} d t \int_{a}^{b}|t-u| e^{t} d t
$$

Summing this for all $i$, we obtain

$$
\sum_{i=1}^{k} \int_{a}^{c_{i}} e^{t} d t \int_{d_{i}}^{b} e^{t} d t \leq \frac{1}{\varepsilon \ln 2} \int_{J_{3}} e^{t} d t \int_{a}^{b}|t-u| e^{t} d t
$$

Since every point of $J_{1}$ and every point of $J_{2}$ are separated by at least one of the intervals $\left(c_{i}, d_{i}\right)$, we have

$$
\sum_{i=1}^{k} \int_{a}^{c_{i}} e^{t} d t \int_{d_{i}}^{b} e^{t} d t \geq \int_{J_{1}} e^{t} d t \int_{J_{2}} e^{t} d t
$$

This proves the theorem. 
Let $K$ be an arbitrary convex body and let, for each $x \in K, \chi(x)$ denote the length of the longest segment contained in $K$ with midpoint $x$. (Equivalently, $\chi(x)$ is the diameter of $K \cap(2 x-K)$.) Define

$$
\chi(K)=\frac{1}{\operatorname{vol}(K)} \int_{K} \chi(x) d x .
$$

Theorem 5.3. For every convex body $K$,

$$
\psi(K) \geq \frac{1}{\chi(K)} .
$$

Proof. We prove, for every partition $K=K_{1} \cup K_{2} \cup K_{3}$ of $K$ into three measurable sets such that the distance of $K_{1}$ and $K_{3}$ is $\varepsilon>0$, the inequality

$$
\operatorname{vol}\left(K_{1}\right) \operatorname{vol}\left(K_{2}\right) \leq \frac{1}{\varepsilon} \operatorname{vol}\left(K_{3}\right) \int_{K} \chi(x) d x
$$

Similarly as before, Theorem 2.7 can be applied to reduce the problem to an assertion about one-dimensional integration: for every interval $[a, b]$ on the line, and every partition $[a, b]=J_{1} \cup J_{2} \cup J_{3}$ into three measurable sets $\left(J_{1}, J_{2}\right.$ are closed, $J_{3}$ is open in $[a, b])$ such that the distance of $J_{1}$ and $J_{2}$ is at least $\varepsilon$,

$$
\int_{J_{1}} e^{t} d t \int_{J_{2}} e^{t} d t \leq \frac{1}{\varepsilon} \int_{J_{3}} e^{t} d t \int_{a}^{b} \min (t-a, b-t) e^{t} d t
$$

Again as before, it suffices to prove this in the case when $J_{3}$ is a single interval $(c, c+\varepsilon)$. As before, we evaluate these integrals and simplify to get the inequality

$$
\left(e^{(b-a) / 2}-e^{\varepsilon / 2}\right)^{2} \leq\left(\frac{e^{\varepsilon}-1}{\varepsilon}\right)\left(e^{(b-a) / 2}-1\right)^{2},
$$

which is clearly true.

The two lower bounds on $\psi(K)$ in Theorems 5.1 and 5.3 are not comparable. The first theorem gives $\psi \geq \Omega\left(n^{-1 / 2}\right)$ for every isotropic body. The second theorem gives $\psi \geq \Omega(1)$ for the isotropic ball, but only the trivial $\psi \geq \Omega(1 / n)$ for the isotropic simplex.

We present below an upper bound on $\psi(K)$. We conjecture that this upper bound is always within a constant factor to the truth. This is equivalent to the conjecture that there is hyperplane cut which is within a constant of the "best" cut.

Let $K$ be a convex body in $\mathbb{R}^{n}$, and let $\alpha(K)$ be the largest eigenvalue of $A(K)$.

Conjecture. $\psi(K)=\Theta(1 / \sqrt{\alpha(K)})$.

Theorem 5.4. For every convex body $K$ in $\mathbb{R}^{n}$,

$$
\psi(K) \leq \frac{10}{\sqrt{\alpha(K)}} .
$$


The proof is immediate from the following lemma, which can be proved either using the localization lemma or (if the best constant is not important) a standard log-concavity argument.

Lemma 5.5. Let $K$ be a convex body in $\mathbb{R}^{n}$ and assume that $b(K)=0$. Let $u$ be any vector in $\mathbb{R}^{n}$ of length 1 , and $\beta:=E_{K}\left(\left(u^{T} x\right)^{2}\right)$. Then

$$
\begin{aligned}
& \operatorname{vol}(K\left.\cap\left\{x: u^{T} x<0\right\}\right) \operatorname{vol}\left(K \cap\left\{x: u^{T} x>0\right\}\right) \\
& \geq \frac{1}{10} \sqrt{\beta} \operatorname{vol}_{n-1}\left(K \cap\left\{x: u^{T} x=0\right\}\right) \operatorname{vol}(K) .
\end{aligned}
$$

We note that it could also be shown easily along the same lines that cuts by hyperplanes do not provide a counterexample to the conjecture.

\section{References}

D. Applegate and R. Kannan (1990): Sampling and integration of near log-concave functions, Proc. 23th ACM Symposium on the Theory of Computing, pp. 156-163.

J. Bokowski (1980): Ungleichungen für des Inhalt von Trennflächen, Arch. Math. 34, 84-89.

J. Bokowski and E. Spencer Jr. (1979): Zerlegung Konvexen Körperdurch minimale Trennflächen, J. Reine Angew. Math. 311-312, 80-100.

C. Borell (1975): The Brunn-Minkowski inequality in Gauss spaces, Invent. Math. 30, 207-216.

J. Bourgain (1991): On the Distribution of Polynomials on High Dimensional Convex Sets, Lecture Notes in Mathematics, Vol. 1469, Springer-Verlag, Berlin, pp. 127-137.

A. Dinghas (1957): Über eine Klasse superadditiver Mengenfunktionale von BrunnMinkowski-Lusternik-schem Typus, Math. Z. 68, 111-125.

M. Dyer and A. Frieze (1992): Computing the volume of convex bodies: a case where randomness provably helps, in: Probabilistic Combinatorics and Its Applications (ed. B. Bollobás), Proceedings of Symposia in Applied Mathematics, Vol. 44, American Mathematical Society, Providence, RI, pp. 123-170.

M. Dyer, A. Frieze, and R. Kannan (1989): A random polynomial time algorithm for approximating the volume of convex bodies, Proc. 21st ACM Symposium on Theory of Computing, pp. 375-381.

M. Gromov and V. D. Milman (1984): Brunn theorem and a concentration of volume of convex bodies, GAFA Seminar Notes, Tel Aviv University.

D. Hensley (1980): Slicing convex bodies and bounds on the slice area in terms of the body's covariance, Proc. Amer. Math. Soc. 79, 619-625.

F. John (1948): Extremum problems with inequalities as subsidiary conditions, in: Studies and Essays Presented to R. Courant, Interscience, New York, pp. 187-204.

A. Karzanov and L. G. Khachiyan (1991), On the conductance of order Markov chains, Order 8, 7-15.

L. Lovász and M. Simonovits (1990): Mixing rate of Markov chains, an isoperimetric inequality, and computing the volume. Proc. 31st IEEE Symposium on Foundations of Computer Science, pp. 346-355.

L. Lovász and M. Simonovits (1993): Random walks in a convex body and an improved volume algorithm, Random Structures Algebra 4, 359-412. 
V. D. Milman and A. Pajor (1989): Isotropic position and inertia ellipsoids and zonoids of the unit ball of a normed $n$-dimensional space, in: Geometric Aspects of Functional Analysis (eds. J. Lindenstrauss and V. D. Milman), Lecture Notes in Mathematics, Vol. 1376, Springer-Verlag, Berlin, pp. 64-104.

A. Prékopa (1971): Logarithmic concave measures with applications to stochastic programming, Acta Sci. Math. (Szeged.) 32, 301-316.

G. Sonnevend (1989): Applications of analytic centers for the numerical solution of semi-infinite, convex programs arising in control theory, DFG Report No. 170/1989, Institut für angew. Mathematik, University of Würzburg.

Received April 11, 1994, and in revised form September 20, 1994. 